\title{
Tomographic Femtosecond X-Ray Diffractive Imaging
}

\author{
K. E. Schmidt, ${ }^{1}$ J. C. H. Spence, ${ }^{1}$ U. Weierstall, ${ }^{1}$ R. Kirian, ${ }^{1}$ X. Wang, ${ }^{1}$ D. Starodub,${ }^{1}$ H. N. Chapman, ${ }^{2}$ \\ M. R. Howells, ${ }^{3}$ and R. B. Doak ${ }^{1}$ \\ ${ }^{1}$ Department of Physics, Arizona State University, Tempe, Arizona 85287-1504, USA \\ ${ }^{2}$ CFEL, DESY, Notkestraße 8522607 Hamburg, Germany \\ ${ }^{3}$ ESRF, BP 220, 38043 Grenoble Cedex 9, France
}

(Received 9 June 2008; published 12 September 2008)

\begin{abstract}
A method is proposed for obtaining three simultaneous projections of a target from a single radiation pulse, which also allows the relative orientation of successive targets to be determined. The method has application to femtosecond x-ray diffraction, and does not require solution of the phase problem. We show that the principal axes of a compact charge-density distribution can be obtained from projections of its autocorrelation function, which is directly accessible in diffraction experiments. The results may have more general application to time resolved tomographic pump-probe experiments and time-series imaging.

DOI: 10.1103/PhysRevLett.101.115507

PACS numbers: 61.05.cp, 82.53.Ps, 87.64.Bx
\end{abstract}

Unless crystallographic redundancy can be taken advantage of, radiation damage provides a well established limit to resolution for imaging in biology. X-ray microscopy of proteins is limited in this way to $10 \mathrm{~nm}$ [1]. Since dose depends on the inverse fourth power of resolution, a severe penalty attends any attempt to improve resolution beyond this barrier, which occurs when the required dose needed to distinguish adjacent image voxels with statistical significance exceeds the damage limit at that resolution (voxel size).

It has been suggested that the development of the freeelectron x-ray laser (FEL) may break this nexus between dose and resolution [2], if it can provide sufficient photons for a useful diffraction pattern in a single pulse, which terminates prior to any of the characteristic times for damage processes. A continuum of such times, femtosecond for electrons, hundreds of femtoseconds for nuclear motion, is associated with the various irreversible damage mechanisms and excitations. Theory suggests that pulses shorter than the Auger decay time of a few femtoseconds may terminate before significant disruption of the valence electron distribution occurs. Experimental evidence, using a $25 \mathrm{fs}$ pulse of soft $(30 \mathrm{~nm}) \mathrm{x}$ rays, now exists for this process of "diffraction-before-destruction" at low $(90 \mathrm{~nm})$ [3] and higher periodically averaged [4] resolution. Since the FEL generates in excess of $10^{12}$ fully coherent photons in such a pulse, the method of diffractive lensless imaging [5], in which real-space images are reconstructed computationally from these scattering patterns, would appear to provide a means of overcoming the radiation damage barrier to high resolution imaging in biology [6]. The shortest FEL wavelength is currently $7 \mathrm{~nm}$ at the Hamburg FLASH facility. Shorter wavelength FELs are being planned and constructed at other sites around the world.

The subsequent destruction of the sample following the initial elastic scattering event, however, has precluded the possibility of three-dimensional (tomographic) imaging of unique structures. To overcome this limitation, we suggest here a means to determine the relative orientation of successive targets.

Two possible arrangements are shown in Figs. 1 and 2. In Fig. 1, a beam splitter and reflecting crystals direct three orthogonal beams onto a nonperiodic target particle producing three far-field diffraction patterns prior to destruction of the target. All three two-dimensional patterns are readout after each x-ray pulse, whereupon a new, identical target such as a biomolecule is inserted in a new orientation.

We will show that the relative orientation of successive targets can be determined even if the structure of the target is unknown.

Following related work by Smilgies on crystals [7], we propose experimental determination of the principal axes of the molecule to describe its orientation relative to the laboratory frame defined by the incident probe beams.

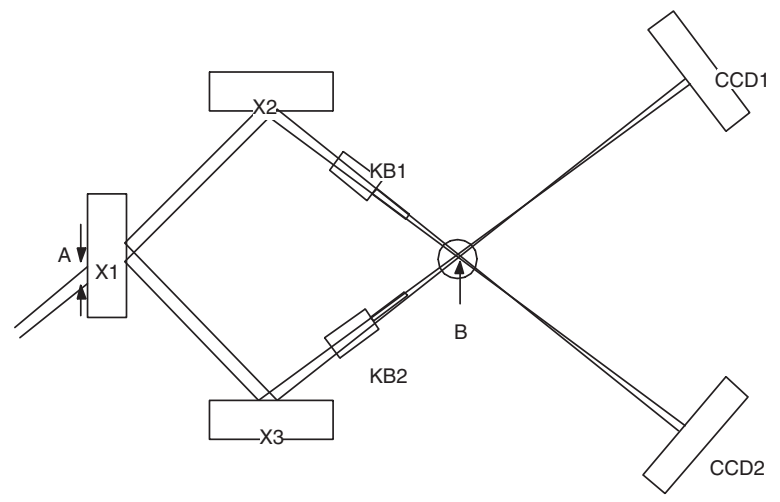

FIG. 1. Scheme for tomographic femtosecond diffraction, drawn for only two beams for simplicity. Beam splitter $X 1$ is set to the dynamical 3-beam diffraction condition. Crystals $X 2$ and $X 3$ operate at the 2-beam dynamical condition. KB1 and $\mathrm{KB} 2$ are focusing optics for the target at $B$, with area detectors CCD1 and CCD2. 


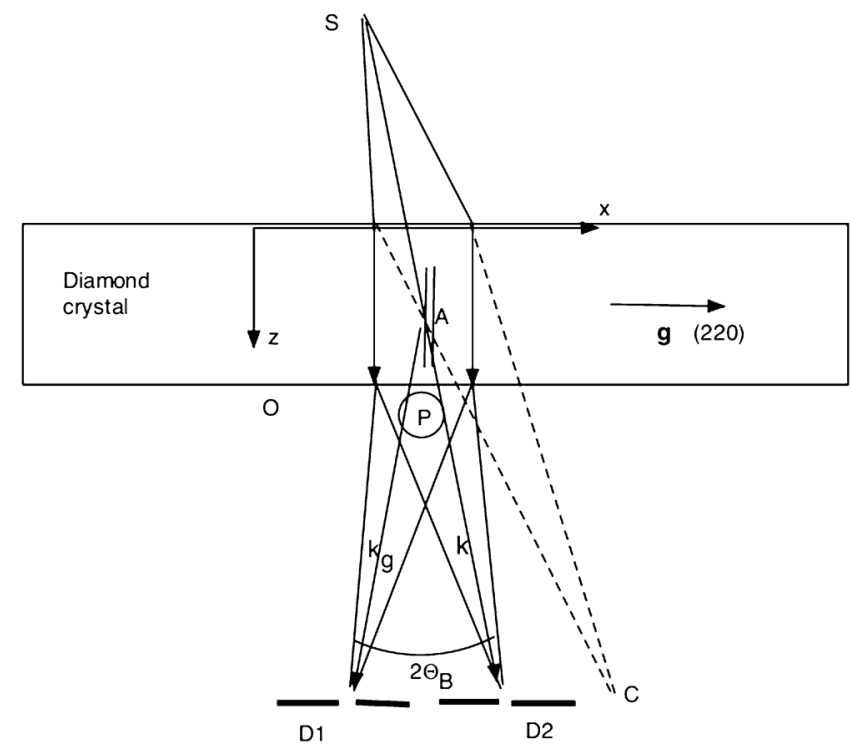

FIG. 2. Two-beam beam splitter with sample shown at $P$ lying on the exit face of the beam splitter. The source $S$ is focused onto two area detectors D1 and D2 containing central beam-dump holes. The two vertical arrows show the direction of the Poynting vector. Three such orthogonal diffracted beams, rather than the two shown, are proposed in the text.

Multiple scattering will be neglected, i.e., the first Born approximation is assumed valid, so that the patterns have inversion symmetry and the target density is a real function.

To expose the principle of the method, assume that the phase problem can be solved by, for example, iterative methods (see [8] for a review). (We will relax this assumption later.) Then, in the "flat" Ewald sphere approximation, i.e., at high probe energy, each beam delivers a projection in real space (along the direction of the corresponding beam) of the scattering strength per unit volume. The projections will be referred to different (randomly positioned) origins, and both enantiomorphs (related by inversion symmetry) will be present with equal likelihood. However once a particular enantiomorph is chosen for one projection, the resulting two-dimensional envelope will constrain the choice of enantiomorph for the other two projections.

Consider the moments of the mass density $\rho(\boldsymbol{r})$ for the target [9]. The zeroth moment delivers the total mass, the first moment delivers the center of mass vector, and the second moment delivers the moment of inertia tensor. By diagonalizing the latter, the principal axes of the target may be found and hence its orientation relative to the lab frame. Taking the center of mass position as the origin, the inertia tensor is

$$
\boldsymbol{I}=\iiint \rho(\boldsymbol{r})\left(r^{2} \boldsymbol{E}-\boldsymbol{r} \boldsymbol{r}\right) d \boldsymbol{r}
$$

where $\boldsymbol{E}$ is the unit tensor and $\boldsymbol{r} \boldsymbol{r}$ is the outer product of the position vector with itself. As with any symmetric tensor, $\boldsymbol{I}$ has only six independent elements, real eigenvalues, and orthogonal eigenvectors corresponding to different eigenvalues.

Now take $\rho(\boldsymbol{r})$ to be the electronic charge density of the target, whose projections in three orthogonal directions are provided by the phased data, and which define the $x, y$, and $z$ directions specified by unit vectors $\boldsymbol{e}_{i}$ in the lab reference frame. The six independent elements of this charge density "inertia" tensor then have the form

$$
\begin{aligned}
I_{z z} & =\iint \rho_{z}(x, y)\left(x^{2}+y^{2}\right) d x d y \\
I_{x y} & =-\iint \rho_{z}(x, y) x y d x d y
\end{aligned}
$$

and similarly for $I_{x x}, I_{y y}, I_{y z}$, and $I_{x z}$. Here $\rho_{\alpha}$ is the projected density along the $\alpha$ direction. Two of these six tensor elements can be computed from each of the three projections, e.g., $I_{z z}$ and $I_{x y}$ from the projection along the $z$ axis. Hence the inertia tensor of the target is fully specified by computing moments and products of inertia from the three projections. This charge density "inertia" tensor will differ from that based on mass but serves equally well to provide a consistent set of principal axes fixed to the molecule and defining alignment. Being symmetric, this tensor may be diagonalized by solving the eigenvalue equations

$$
\boldsymbol{I} \cdot \boldsymbol{B}=b \boldsymbol{B}
$$

for the three eigenvalues $b$ and corresponding eigenvectors $\boldsymbol{B}$. These eigenvectors define a new orthogonal coordinate system $\boldsymbol{e}_{j}^{\prime}$ in which the three unit vectors lie along the principal axes of the inertia tensor. Barring degeneracy among the eigenvalues, the three eigenvectors are unique to within a sign, and therefore offer a natural means of specifying the orientation of the target relative to the incident beam directions (lab frame). With the unit vectors $\boldsymbol{e}_{i}$ of the lab frame and $\boldsymbol{e}_{j}^{\prime}$ both known, the angles between the principal axes of the target and the lab frame can immediately be computed. Thus the orientation of the target has not only been defined by introducing the principal axes of the inertia tensor, but also specified (within polarity) relative to the lab coordinates.

Clearly, then, to establish the orientation of the particle it suffices to (i) record three diffraction patterns, one for each of the three incident beam directions, (ii) invert the diffraction patterns using phase retrieval techniques to yield three real-space projections of the scattering strength, (iii) compute the first moment of each projection to obtain the center of mass position for that projection (iv) compute the second order moments of each projection (products of inertia) about the center of mass to obtain one diagonal and one off-diagonal tensor element, (v) diagonalize the resulting tensor to obtain the eigenvectors of the tensor, (vi) compute the orientation of each beam relative to the 
eigenvectors of the target in order to determine the angles between laboratory and principal axes coordinates. (vii) If this process is repeated for many successive identical targets in random orientations, their relative orientations can be found, and hence a complete three-dimensional tomographic image can be assembled by standard tomographic techniques such as filtered backprojection.

We now extend this analysis to show that the principal axes may be found, even without solving the phase problem, by working with the autocorrelation of the sample density

$$
A(\boldsymbol{r})=\int d \boldsymbol{r}^{\prime} \rho\left(\boldsymbol{r}+\boldsymbol{r}^{\prime}\right) \rho\left(\boldsymbol{r}^{\prime}\right) .
$$

A typical product of inertia is

$$
\begin{aligned}
I_{x y}^{A} & =-\int d \boldsymbol{r} x y A(\boldsymbol{r})=-\int d \boldsymbol{r}^{\prime} \rho\left(\boldsymbol{r}^{\prime}\right)\left[\int d \boldsymbol{r} x y \rho\left(\boldsymbol{r}+\boldsymbol{r}^{\prime}\right)\right] \\
& =\int d \boldsymbol{r}^{\prime} \rho\left(\boldsymbol{r}^{\prime}\right)\left[I_{x y}^{\rho}-x^{\prime} y^{\prime} M\right]=2 M I_{x y}^{\rho},
\end{aligned}
$$

where $M=\int d \boldsymbol{r} \rho(\boldsymbol{r})$, and with use of the parallel axis theorem to calculate the product of inertia for the shifted coordinates. Therefore, the principal axes of the autocorrelation function are the same as the principal axes of the corresponding density.

Given a "flat" Ewald sphere, the Fourier transform of each diffraction pattern (intensity) directly provides a projection of the three-dimensional autocorrelation function of the density, and the analysis simply requires changing $\rho(\boldsymbol{r})$ to $A(\boldsymbol{r})$ in Eq. (2).

More generally, the moment of inertia can be calculated from the second derivative of the Fourier transform. Denoting these by a tilde,

$$
\begin{aligned}
\tilde{\boldsymbol{\rho}}(\boldsymbol{q}) & =\int d \boldsymbol{r} e^{-i \boldsymbol{q} \cdot \boldsymbol{r}} \rho(\boldsymbol{r}), \\
\tilde{A}(\boldsymbol{q}) & =\tilde{\rho}(\boldsymbol{q}) \tilde{\boldsymbol{\rho}}(-\boldsymbol{q}) \\
I_{x y}^{A} & =\left.\partial_{q_{x}} \partial_{q_{y}} \tilde{A}(\boldsymbol{q})\right|_{\boldsymbol{q}=0}=\left.\partial_{q_{x}} \partial_{q_{y}} \tilde{\rho}(\boldsymbol{q}) \tilde{\boldsymbol{\rho}}(-\boldsymbol{q})\right|_{\boldsymbol{q}=0} \\
& =\left.\tilde{\rho}(0)\left[2 \partial_{q_{x}} \partial_{q_{y}} \tilde{\rho}(\boldsymbol{q})\right]\right|_{\boldsymbol{q}=0}=2 M I_{x y}^{\rho}
\end{aligned}
$$

as before. Replacing $\tilde{A}\left(q_{x}, q_{y}, 0\right)$ (flat Ewald sphere) with the correct Ewald sphere (finite radius) diffraction pattern, and taking the incident wave vector $\boldsymbol{k}$ along $z$,

$$
\begin{aligned}
& \left.\partial_{q_{x}} \partial_{q_{y}} \tilde{A}\left(q_{x}, q_{y}, \sqrt{k^{2}-q_{x}^{2}-q_{y}^{2}}-k\right)\right|_{q_{x}=q_{y}=0} \\
& \quad=\left.\partial_{q_{x}} \partial_{q_{y}} \tilde{A}\left(q_{x}, q_{y}, 0\right)\right|_{q_{x}=q_{y}=0}
\end{aligned}
$$

so that the moments of the Fourier transform of the diffracted data still yield the same principal axes.

We have investigated this procedure using detailed numerical simulations based on data in the Protein Database for GroEL-GroES protein complex (PDB entry 1SVT). The three-dimensional density was synthesized from the tabulated atomic coordinates. Figure 3 shows the projected
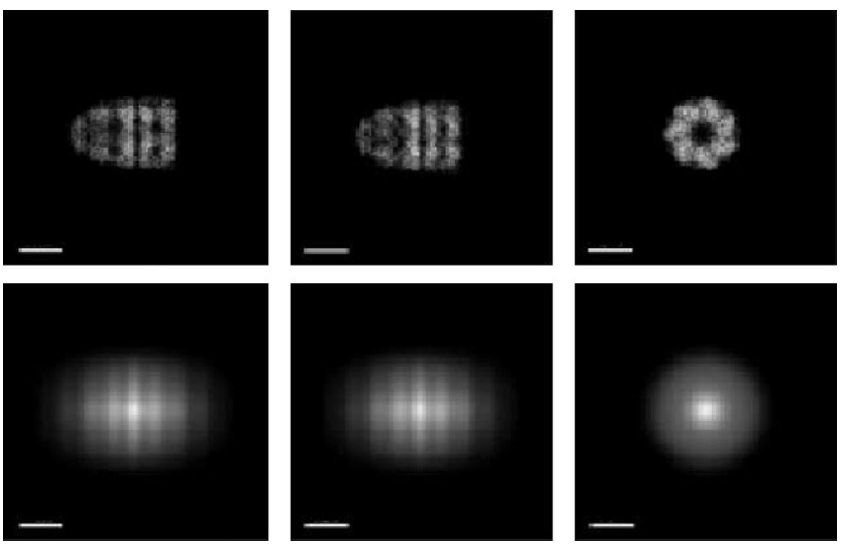

FIG. 3. The three orthogonal projections of the GroEL charge density (upper) and the corresponding projections of the autocorrelation function (lower). The bar indicates $10 \mathrm{~nm}$.

densities and corresponding projected autocorrelation functions using the principal axes obtained from Eq. (2). A second density was then generated in a random orientation with respect to the first, as shown in Fig. 4. For each of these orientations the principal axes were determined using both the densities and the autocorrelation functions, giving similar results. The rotation matrix needed to rotate from the first (Fig. 3) to the second (Fig. 4) orientation was generated from the principal axes. As a result of inversion symmetry there are four distinct choices of rotation matrix (corresponding to choices of eigenvector signs) when the autocorrelation function is used. The correct rotation matrix was obtained by testing each to see which predicted lines of intensity in diffraction patterns common to two orientations. (Any two planes in reciprocal space passing through the origin must intersect along a common line.) In this way only one rotation matrix will be found to give consistent results. Numerical trials have found this procedure to be reliable with several different test objects. Our use of specific common lines should be more robust for noisy data than common line search methods. (Shneerson
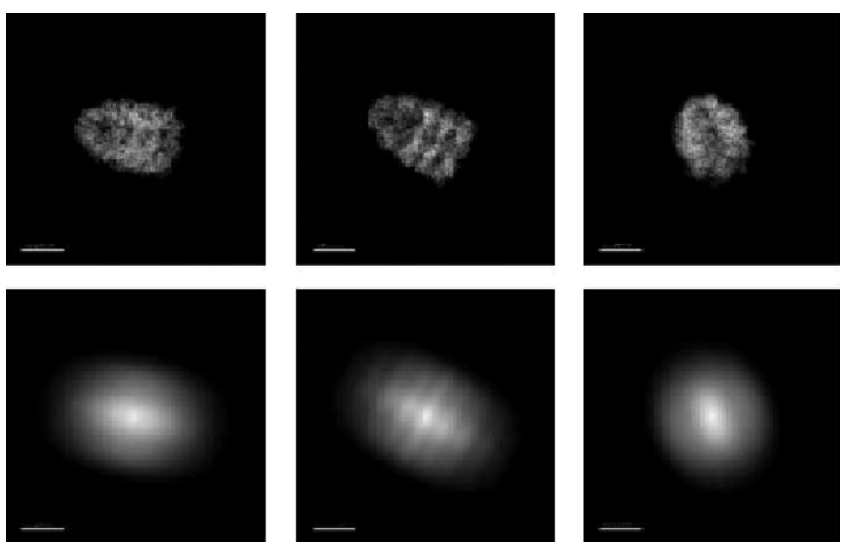

FIG. 4. Projections of GroEL density (upper) and autocorrelation function (lower) in a second random orientation. 
et al. [10] have shown that identifications of common lines in diffraction pattern down to a mean photon count of 10 per pixel enables the determination of their relative orientations without the need to solve the phase problem.)

This treatment easily extends to the case where the beams from the beam splitter are not orthogonal. Reciprocal vectors can be defined in the usual way, so that each pattern lies in the plane of two of these vectors. The products of inertia may be simply evaluated in terms of these reciprocal vectors, and finally transformed into the required lab frame moments.

Two separate experimental implementations of this approach are suggested in Figs. 1 and 2, each with an incident beam close to the [111] direction of a diamond beam splitter crystal, set to simultaneously excite the [022] and [2220] reflections. For an x-ray energy close to $7 \mathrm{keV}$, this generates three orthogonal beams with Bragg angles of $45^{\circ}$. The Borrmann effect [11] may then be used to produce three beams of approximately equal intensity for a crystal thickness of order $1 \mathrm{~mm}$, due to 3-beam multiple scattering [12-14]. This remarkable effect, in which wave field components with zero crossings at atom positions avoid photoelectron production, reduces absorption in the beam splitter by many orders of magnitude. Crystals $X 1$ and $X 2$ operate at 2-beam dynamical conditions, for which reflectivities of greater than $90 \%$ are possible. For the arrangement in Fig. 1, the important experimental challenge is to generate three incident beams that converge focused onto a micron-sized volume of space at the same instant in time. "Diffraction before destruction" will require pulse durations of $10 \mathrm{fs}$ or less, corresponding to a spatial pulse length of at most $3 \mu \mathrm{m}$, a technically challenging but feasible length scale for experimental realization. [Calculations show that $8 \mathrm{keV}$ x-ray pulses reflected from $\mathrm{Si}(111)$ are stretched by about 4 fs [15].]

A monolithic integration of this arrangement may be possible. The arrangement in Fig. 2, with sample mounted on the beam splitter, provides isochronal, but unfocused, optical paths to the sample. This arrangement is better suited to long exposures of continuous radiation for stationary samples. We do not provide detailed phase-space matching calculations here, but note that the beam divergence of one planned FEL, the Linac Coherent Light Source at Stanford, California, is $1.1 \times 10^{-6} \mathrm{rad}$, which is less than a typical perfect-crystal rocking curve width of $3.4 \times 10^{-5} \mathrm{rad}$. An energy spread of $\Delta E / E=1.4 \times 10^{-4}$ can be expected after monochromation at $8 \mathrm{kV}$, with a beam width of $20 \mu \mathrm{m}$. Estimates suggest that even with less than one scattered photon per pixel, phasing and reconstruction is possible $[10,16,17]$.

We conclude that a determination of the relative orientation between successive particles of unknown structure (each initially in a random unknown orientation with respect to the laboratory frame) may be achieved without the need to solve the phase problem.
For a stream of identical molecules in random orientations, this would allow data from different molecules to be merged in the correct relative orientation. After phasing the resulting three-dimensional reciprocal-space data, a tomographic image can then be reconstructed. We have also shown that the orientation of successive objects can be determined from autocorrelation functions, so that a solution of the phase problem is not required. The entire procedure cannot distinguish enantiomorphs. Stereoscopic projections might be obtained from just two projections. We have suggested experimental implementations for this method for femtosecond $\mathrm{x}$-ray diffraction. This analysis applies to any penetrating particles (e.g., neutrons or high energy electrons), insofar as the scattering can be characterized by a scalar potential and the orientation and structure of the sample are unknown (unlike goinometerbased systems where both coordinate systems are known). This might include, for example, the tracking of the orientation of a single body from which nondestructive diffraction patterns can be obtained as function of time. "Proof of principle" measurements at optical wavelengths are currently under way.

This work was supported by NSF IDBR 0555845 and CBST at UC Davis.

[1] M. R. Howells et al., arXiv:physics/0502059v1 [J. Electron Spectrosc. Relat. Phenom. (to be published)].

[2] R. Neutze, R. Wouts, D. van der Spoel, E. Weckert, and J. Hajdu, Nature (London) 406, 752 (2000).

[3] H. N. Chapman et al., Nature Phys. 2, 839 (2006).

[4] S. P. Hau-Riege et al., Phys. Rev. Lett. 98, 145502 (2007).

[5] J. W. Miao, P. Charalambous, J. Kirz, and D. Sayre, Nature (London) 400, 342 (1999).

[6] J.C.H. Spence, in Science of Microscopy, edited by P. Hawkes and J.C. H. Spence (Springer, Berlin, 2007).

[7] D. Smilgies, J. Appl. Crystallogr. 41, 363 (2008).

[8] S. Marchesini, Rev. Sci. Instrum. 78, 049901 (2007).

[9] H. Goldstein, Classical Mechanics (Addison-Wesley, New York, 1950).

[10] V. L. Shneerson, A. Ourmazd, and D. K. Saldin, Acta Crystallogr. Sect. A 64, 303 (2008).

[11] B. W. Batterman and H. Cole, Rev. Mod. Phys. 36, 681 (1964).

[12] M. Umeno and G. Hildebrandt, Phys. Status Solidi A 31, 583 (1975).

[13] M. Umeno and G. Hildebrandt, Acta Crystallogr. Sect. A 31, S253 (1975).

[14] J. M. Zuo, R. Hoier, and J. C. H. Spence, Acta Crystallogr. Sect. A 45, 839 (1989).

[15] S.D. Shastri, P. Zambianchi, and D.M. Mills, J. Synchrotron Radiat. 8, 1131 (2001).

[16] V. Elser, arXiv:0709.3858v1.

[17] A. Ourmazd and D. Saldin (private communication). 